\title{
Development of quadruped walking robot with spherical shell: improvement of climbing over a step
}

\author{
Takeshi Aoki ${ }^{*}$, Keisuke Asami, Satoshi Ito and Shotaro Waki
}

\begin{abstract}
We have proposed and developed a new quadruped walking robot with a spherical shell, called "QRoSS". QRoSS is a transformable robot that can store its legs in the spherical shell. The shell not only absorbs external forces from all directions, but also improves mobile performance because of its round shape. In rescue operations at a disaster site, carrying robots into a site is dangerous for operators because doing so may result in a second accident. If QRoSS is used, instead of being carried in, robots are thrown in, making the operation safe and easy. We developed the QRoSS series and conducted basic experiments to verify performance, which includes landing, rising and walking through a series of movements. This paper reports details of a mechanical design to improve walking performance and development of the QRoSS V prototype model. We discuss the rising operation and performance of climbing over a high vertical step.
\end{abstract}

\section{Introduction}

Rescue robots have recently been developed as a way to gather information on rough terrain environments, such as disaster sites where it is difficult for people to enter. Some examples are leg types such as the BigDog [1] and the LS3 [2], infinite rotation types such as vehicles composed of wheels like the HANZO [3], or tacked belts like the Quince [4], the Souryu-III [5] and the iRobot 510 PackBot [6], and hybrid types like the Titan X [7]. However, the rescue robots have a problem in that they must travel from a safe area to the investigation sites. This uselessly consumes limited power resources, thereby shortening operation time. Moreover, if rescue operators carry the robots to the investigation site themselves, their risk of accident and injury increases. If robots can be thrown into an uneven terrain from a safe area, consumption of electricity for travel is decreased, and operators will be safe with no need to enter the danger zone. These robots are called "Throwable robots."

\footnotetext{
*Correspondence: takeshi.aoki@it-chiba.ac.jp

Department of Advanced Robotics, Chiba Institute of Technology, 2-17-1 Tsudanuma, Narashino 257-0016, Chiba, Japan
}

Example throwable robots are the iRobot 110 First Look [8], which is a small crawler robot with two flipper arms and Sand Flea [9] created by Boston Dynamics, a small wheel type robot equipped with a jumping mechanism to fly over high steps using gas power. Many throwable robots have been developed as wheel-type or track belt type; our motivation is to develop them with walking mobility. Legged robots can discretely choose contact points on the ground by statically maintaining stability with the remaining three legs while one leg swings. The static walk is important for traveling on crumbling rubble because robots can prevent secondary disasters without collapsing the rubble, and even dynamic walk cannot climb a high step on a vertical wall. Further, with the use of sensors, it is easy to create a stable posture for searching.

We have been researching and developing transformable legged robots "QRoSS: Quadruped Robots with Spherical Shell" series [10,11], which have a spherical outer shell and feature walking mode and shock-proof mode when landing. They are small and can be thrown in by rescue operators because they have a spherical outer shell that can absorb landing shock (Fig. 1). Also, they are 


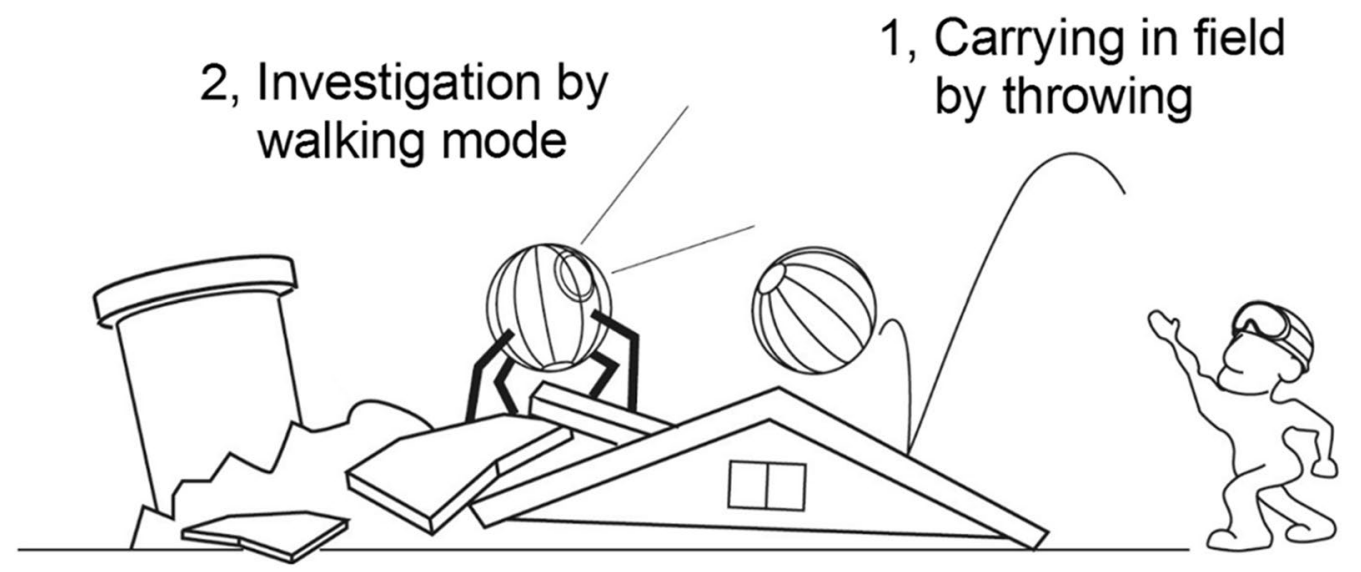

Fig. 1 Application concept of our robot

viable when upside down, so they do not depend on the posture during landing and can turn over autonomously. QRoSS II can do a hybrid motion, which is rotating locomotion using the spherical shell; it is considered more efficient than leg movement on flat terrain [11].

MorpHex III is a transforming Hexapod Robot that can be changed to ball mode, hexapod walking mode and rotational transfer mode by leg actuators and a body actuator [12]. However, because the ball shape is formed by the leg mechanisms, it cannot absorb impact. Even if a structure is used in which the outline of the leg mechanisms can receive force, designing it to be lightweight enough for a mobile robot is difficult. We propose making the spherical outer shell and the walking mechanisms independent of each other. Jumping robot [13] has an outer cage and can jump on two legs; the cage can absorb external forces. However, it cannot travel by static walk using its own legs.

When using a walking robot in a rescue operation, the robot must be able to travel on large rubble in uneven terrain. QRoSS II succeeded in climbing over a step of 100 [mm] height, shown in Fig. 2; however, the outer shell interferes with the step when climbing because previous QRoSS series are a vertically symmetrical design and the lower half of the outer shell prevents climbing. The ratio of allowable step height and the diameter of the spherical outer shell is only 34 [\%]; the robot has not been able to climb over a large step with respect to the shell size. We have also looked at solutions with a robot that flies over obstacles by jumping [14]; however, we now consider it difficult to achieve both jumping and walking performance compatibility. Consequently, it is important to improve each performance independently.

We propose and are developing QRoSS V with new mechanisms to improve walking performance without

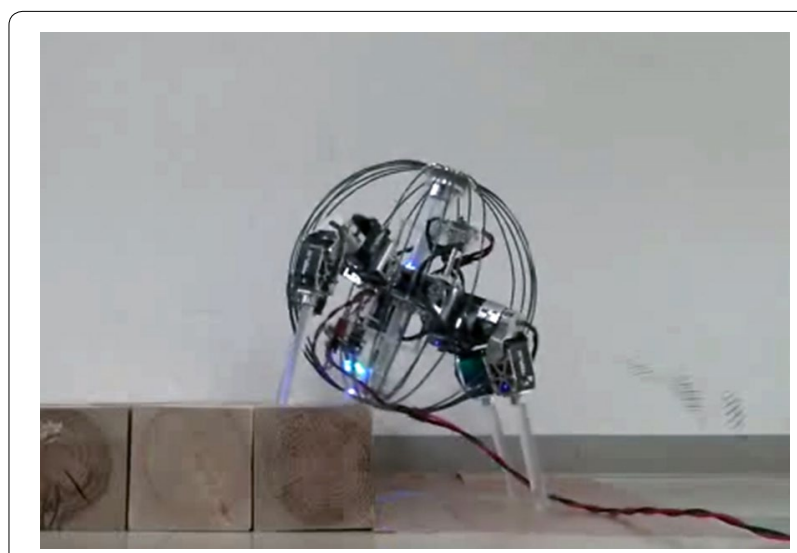

Fig. 2 QRoSS II climbs over step $100 \mathrm{~mm}$ high

increasing its outer shell size; however, we need to rebuild its rising operation. This paper reports development of QRoSS V and analyses of a new rising operation for it and reports on the performance experiment of climbing over a vertical step. The remainder of this paper is organized as follows: Section II gives details of mechanical design of QRoSS V; Section III explains statics analysis of the rising operation, which comprises rising and rolling over motions; Section IV presents and discusses performance experiments.

\section{Design of QRoSS V}

In an irregular environment where rescue robots perform exploratory activities, the robots must be mobile enough to travel on uneven ground and climb over steps. Although the robot can be enabled to get over high steps by enlarging body size and expanding the legs, it becomes so heavy that it may collapse the rubble and the soft 


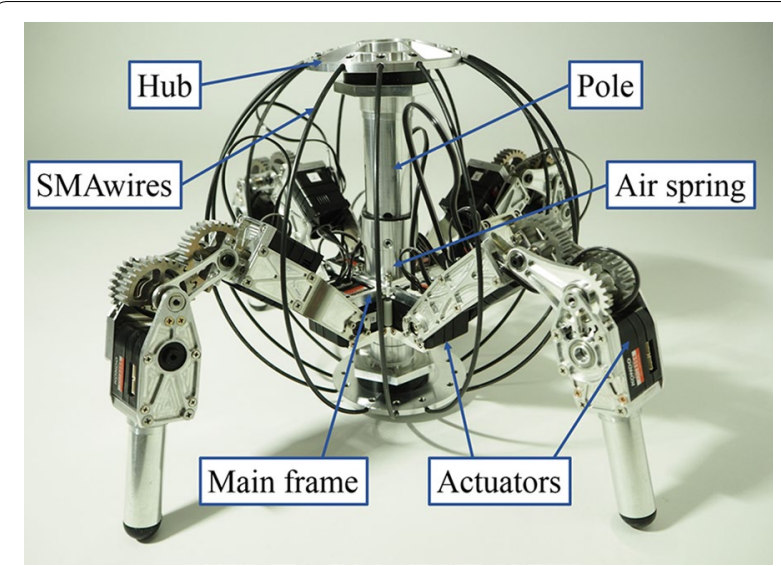

Fig. 3 Overview of QRoSS V

Table 1 QRoSS V Specifications

\begin{tabular}{ll}
\hline Length & $190[\mathrm{~mm}]$ \\
Width & $190[\mathrm{~mm}]$ \\
Height & $197[\mathrm{~mm}]$ \\
Diameter of spherical shell & $200[\mathrm{~mm}]$ \\
Length of leg & $220[\mathrm{~mm}]$ \\
Mass(including battery) & $1295[\mathrm{~g}]$ \\
Active DOF & 12 \\
Battery & LiPo 2 cell 7.4 [V] \\
\hline
\end{tabular}

ground. In this research, we designed a new prototype model "QRoSS V". Compact, lightweight and capable of overcoming large steps, the prototype can perform on uneven terrain for rescue operations. The leg mechanisms were arranged at the center of the outer shell in the previous QRoSS series, and were a vertically symmetrical structure. However, because the legs must be stored in the outer shell, leg length cannot be extended significantly and the ratio of the leg length to the diameter of the outer shell is small. Thus, by offsetting the fixing position of the outer shell and the leg mechanisms from the center, it becomes possible to increase the distance between the outer shell and the ground surface when the legs are expanded from the shell. Further, the legs can be stored in the outer shell by offset at the fixed position, and made long enough to enable large steps.

\section{Overview}

We developed QRoSS V to achieve both high mobility and to be robust against shock. Figure 3 is QRoSS V and Table 1 lists specifications. QRoSS V is composed of the spherical outer shell and four legs, which are arranged radially from the center pole and offset $36.5[\mathrm{~mm}]$ from the center of the shell. Although the Diameter of the

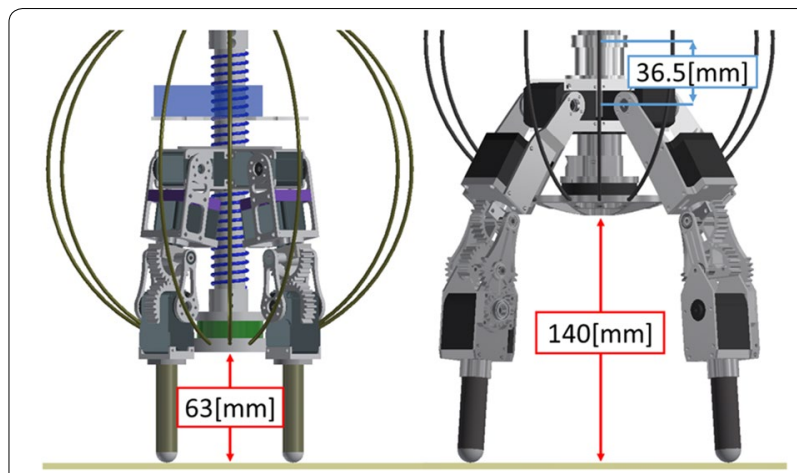

Fig. 4 Comparison of the lower half of the space; Left is QRoSS I and Right is QRoSS V

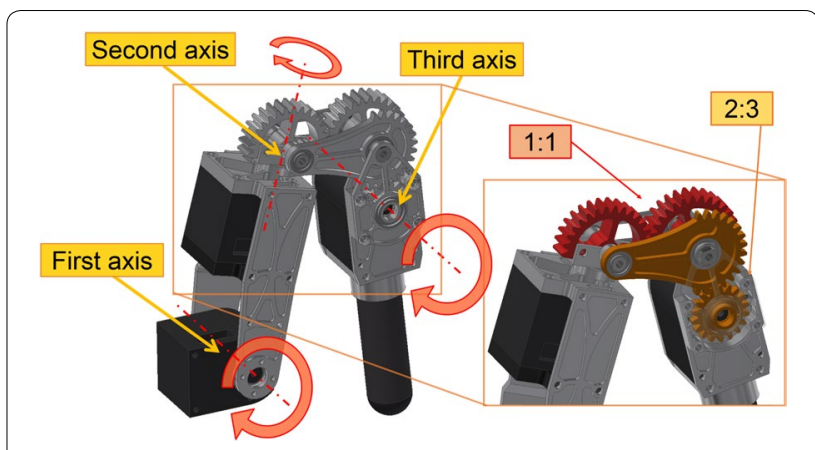

Fig. 5 Leg design of QRoSS V

spherical outer shell is $200[\mathrm{~mm}]$ and the same size as QRoSS I, the distance between the lower side of shell and the ground increases from 63 to 140 [mm] (Fig. 4).

\section{Leg design}

A new leg mechanism is designed based on QRoSS I's mechanism (Fig. 5). Each leg consists of three D.O.Fs; a double joint mechanism using two gears on the third axis achieves a wide working range. However, because the double joint mechanism is a speed up gear mechanism, the output torque of the third joint decreases, limiting the wide range. Thus, we designed a reduction gear mechanism whose ratio is $2: 3$ and added the double joint mechanism; the reduction gear ratio of the third joint could be reduced from 2:1 to $4: 3$. This reduction gear mechanism is designed to be thin, preventing interference with other mechanisms when storing the leg inside the outer shell. Each actuator of the leg joint is the Kondo KRS3304 ICS, a small servo motor with Maximum torque of $13.9[\mathrm{kgf} \mathrm{cm}]$. This has made it possible to extend the leg length beyond that of QRoSS I, thereby making a higher step possible.

As shown in Fig. 6, QRoSS I and V have a wide work range of the leg in the vertical direction. And in QRoSS 


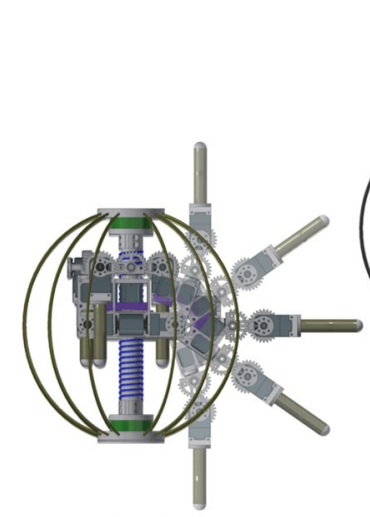

QRoSS I

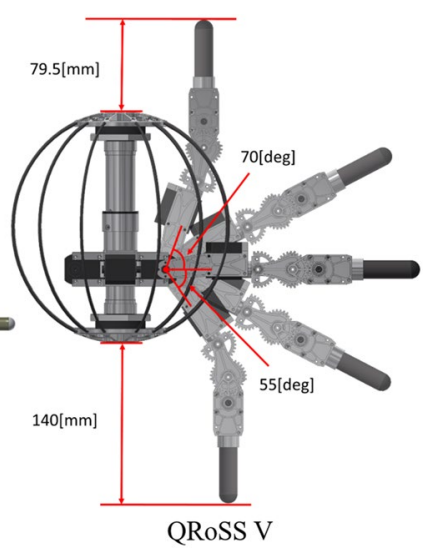

Fig. 6 Comparison of leg work range in vertical direction
$\mathrm{V}$, by offsetting the leg from the center of the spherical outer shell, the length of the leg stored inside the outer shell is extended and the distance between the outer shell and the ground is increased. However, with QRoSS V, when the posture of the body is reversed, the abdominal distance is only $79.5[\mathrm{~mm}]$ at maximum. Although walking motion is considered possible, the mobility is low because the leg work range is smaller than the normal posture. Thus QRoSS V needs a turn over motion to return to the normal state.

\section{Shell design}

The spherical outer shell of QRoSS V, shown in Fig. 7, is composed of a cage with a center pole through the

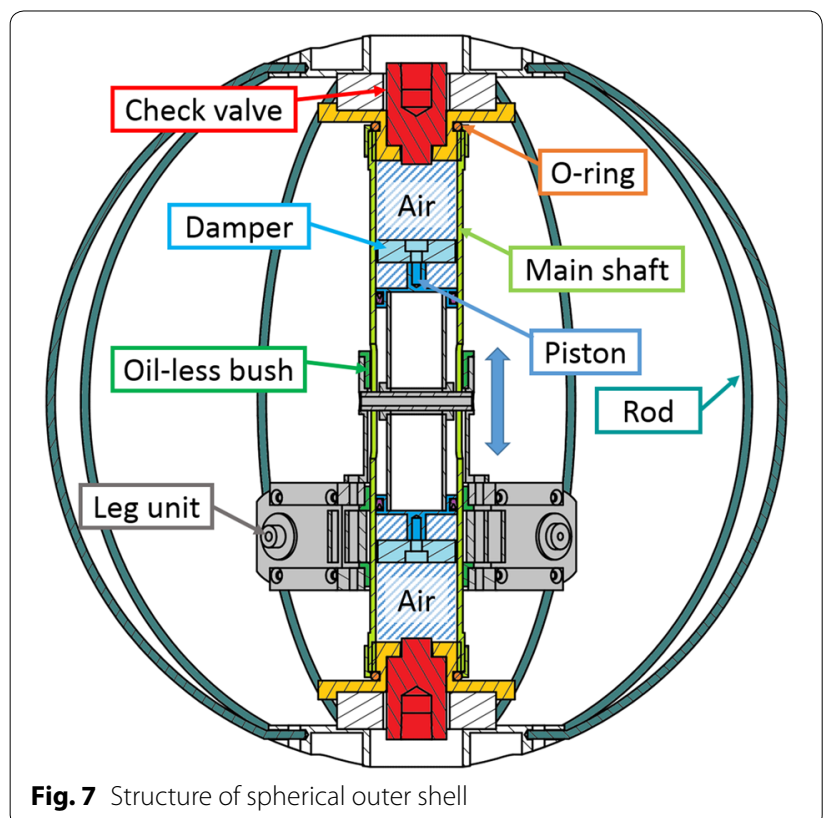

absorbers. The 12 rods of the cage are 2.3 [mm] diameter and super elasticity rods made of titanium alloys. The absorbers, comprised by polyurethane foams, are arranged between the wire hub and the center pole, because an axial direction of the center pole has no modification element. The center frame, the leg's base, is floating on the center pole and mounted by two spring elements; it can slide on the surface and absorb shock in the axial direction. In previous models, although the spring elements were coil springs of steel, they were not lightweight. In the shock absorbing mechanism of QRoSS $\mathrm{V}$, a lightweight air spring increases weight and saves space. The air spring maintains airtightness inside the cylinder, where the internal pressure is increased when the piston moves. This internal pressure creates a force against the piston, making it possible to obtain a restoring force according to the amount the piston moves. It can adjust the restoring force by changing the air pressure inside the cylinder, unlike a mechanical spring. Further, a damper mechanism using the viscous resistance of inside air can be mounted. The center frame and the piston of the air spring are fixed with a shaft, and the Oilesbush is arranged to reduce the sliding resistance between them. Check valves are placed at both ends of the center pole to maintain the internal pressure of the air springs. QRoSS V is designed to absorb the resulting shock when dropped from $2 \mathrm{~m}$. For the maximum deformation of the rods, the impact force is estimated by setting the contact time to $0.01[\mathrm{~s}]$, and the maximum deformation is determined to be 29.6 [mm] from the structural analysis using CAE (Autodesk Inventor). Even if the outer shell is deformed the maximum amount, the legs stored in the outer shell do not contact the ground. The relationship between the internal pressure and the piston stroke of the air spring is determined from the balance between the potential energy and the elastic energy before and after the drop. The mechanical limit of the stroke length is

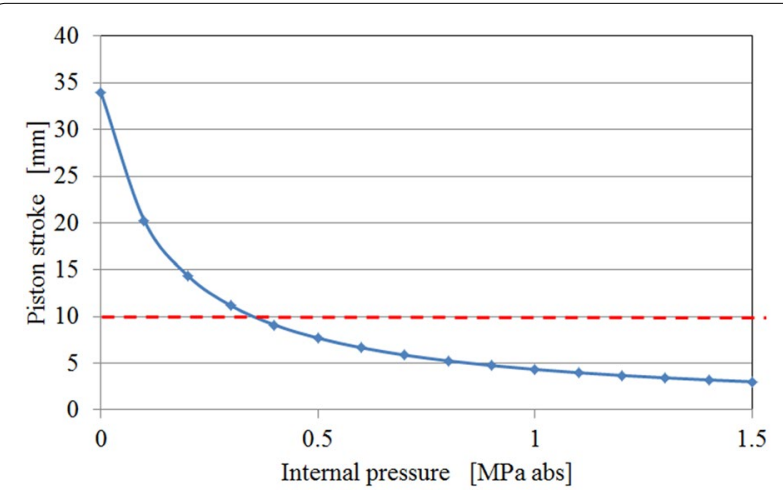

Fig. 8 Air spring internal pressure and stroke required when QRoSS V drops from a 2 [m] height 


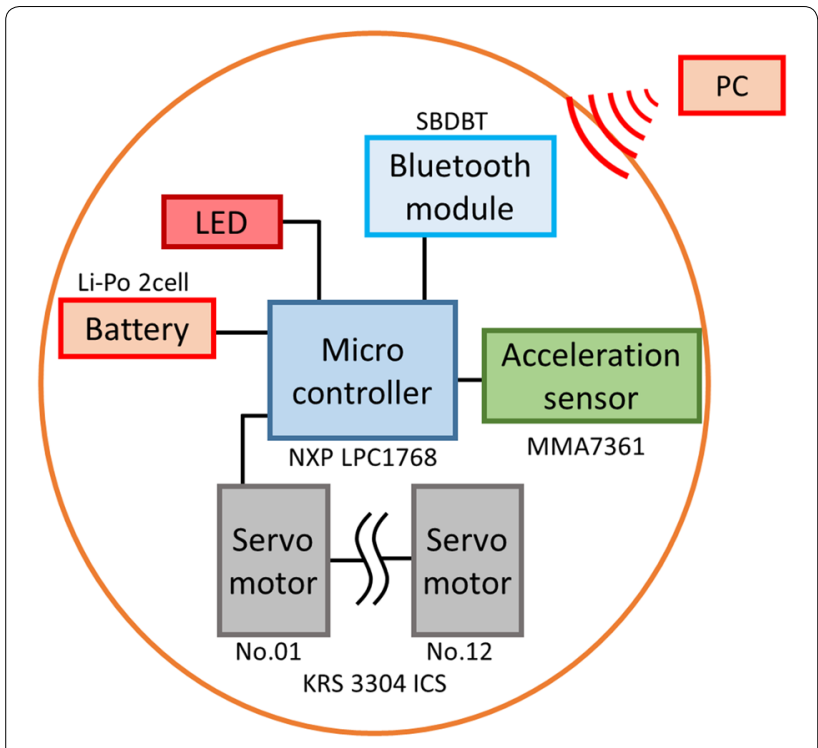

Fig. 9 System configuration

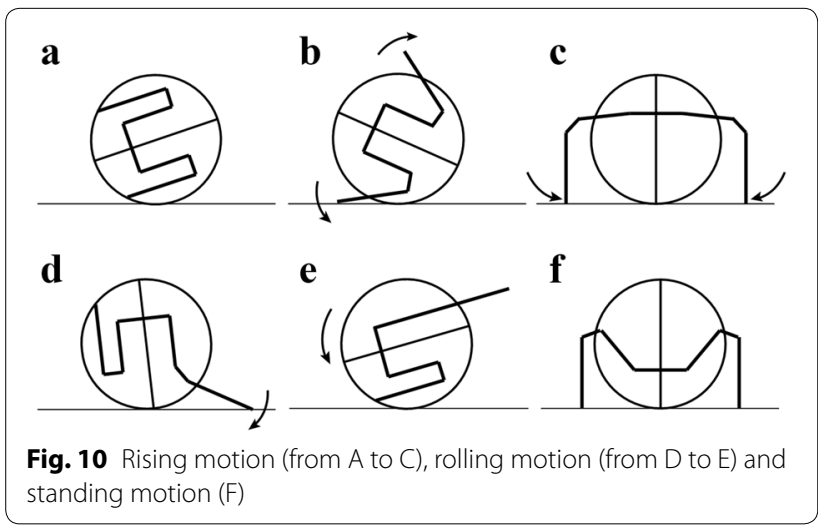

$10[\mathrm{~mm}]$ and its cylinder diameter is 22 [mm]. As Fig. 8 shows, we can adjust its pressure in the range of 0.4 to 1.0 [MPa abs].

\section{System configuration}

Figure 9 shows the system configuration of QRoSS V. We have only done tele-operation because the purpose of this prototype model is to verify mobility. QRoSS V is controlled by one micro controller, which is the mbed NXP LPC1768 with a USB Bluetooth module. These micro controllers produce the paths of the legs and command signals for servo motors of the legs. Inclination of the body is continually detected by the 3 axis accelerometer sensor. The prototype model is operated from a laptop computer using wireless LAN.

\section{Analysis of rising operation}

The QRoSS series demonstrated several motions and verified effectiveness of our design concept [10,11]. Their rising operation was achieved by the motion path of the legs. Because the robot cannot detect the contact point with the ground when it lands on rubble, it needs to rise by motion of the legs from every state. We should divide the rising operation and take into account rising and standing, because all the leg actuators have small outputs; using the spherical outer shell is very effective to roll its body posture. With QRoSS V, Fig. 10 shows its rising operation composed of the rising motion (from A to $\mathrm{C}$ ), rolling motion (from $\mathrm{D}$ to $\mathrm{E}$ ) and standing motion $(\mathrm{F})$. These operations are performed statically. Because they are performed on rubble, there is a risk that rubble will collapse if done dynamically. All legs start to deploy from the outer shell using only the third joint of each leg and the shell starts to rotate as shown in B. After the outer shell rises and its center pole becomes vertical, the rising motion is finished by standing up with a paddle motion by the legs as shown in C. However, this posture is upside down; it now must roll over to the opposite posture. At start of the rolling motion, all legs are stored in the shell once. Next, one leg is deployed and kicks the ground, the spherical shell starts rotating along its shape and all legs start preparing for the next posture, from D to E. F is the standing motion, composed of stopping the shell rotation by toes touching the ground and standing up vertically.

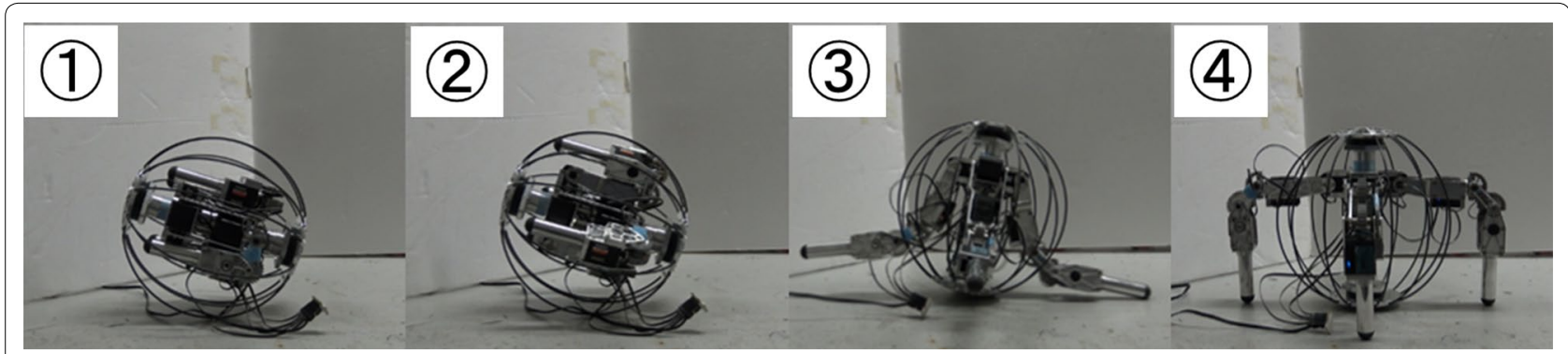

Fig. 11 Experiment of rising motion 


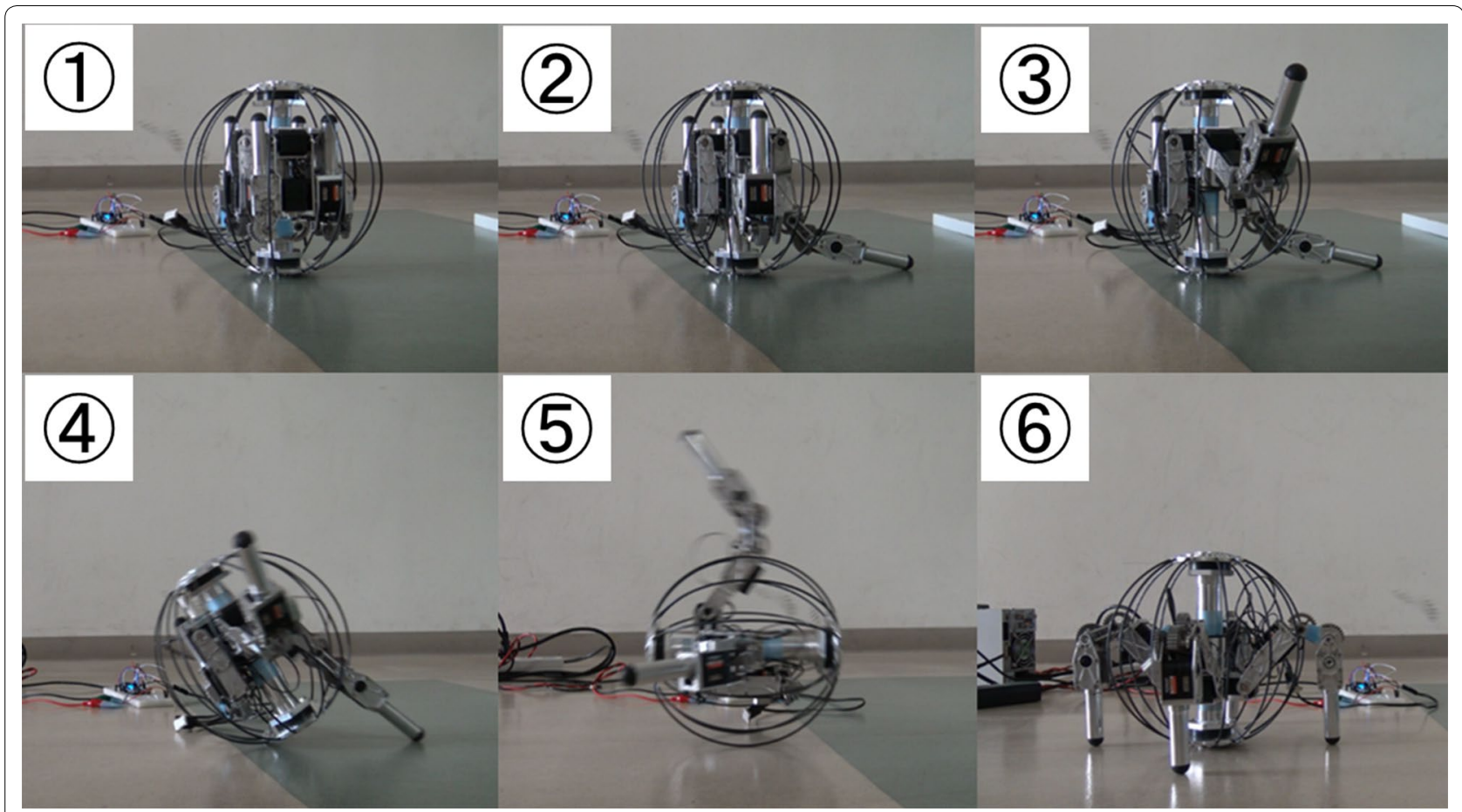

Fig. 12 Experiment of rolling motion

Two experiments confirm the rising motion and the rolling motion of the rising operation from the landing and the store state to standing state. Figure 11 shows the rising operation. The shell starts rotating by kicking at No.2, all legs step to the ground and stop shell rotation from No.3 to No.4. Figure 12 shows rolling operation, where QRoSS V returns from the upside-down posture. One leg is deployed from the shell and prepares to kick the ground, from No.1 to No.3. From No.4 to No.5, the

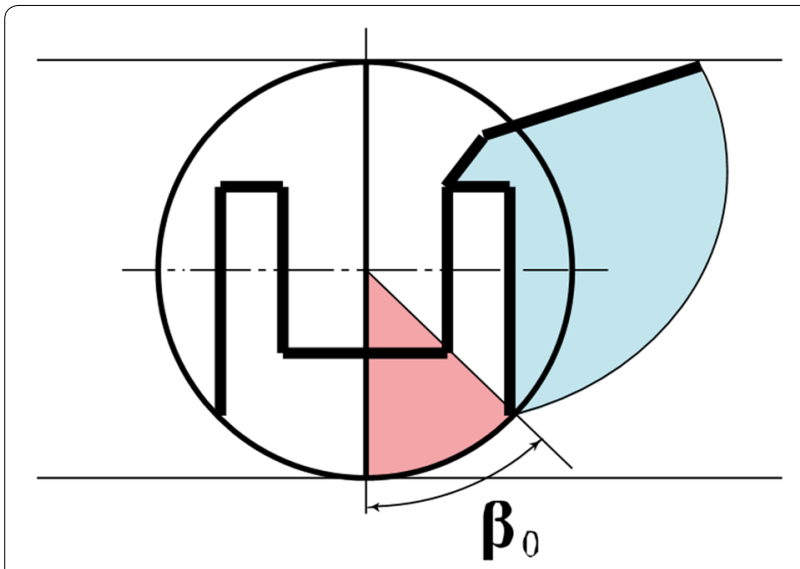

Fig. 13 Direction of rotation shell starts rolling along the curve of the spherical shell by the leg; No.6 is the posture when rolling motion finishes.

Figure 13 shows direction of the first rotation on rising. The blue area is the work range of the third joint with shin. The range of $\beta_{0}$ is not affected by the first deployment of the legs; this value is 51.6 [deg]. If the contact point with the ground is in this area, QRoSS V does not need the rising motion or the rolling motion; it needs only to stand up with the deployed legs, as in F of Fig. 10. However, other areas of $\beta_{0}$ need both motions. QRoSS has to rise not only on flat and horizontal ground, but on

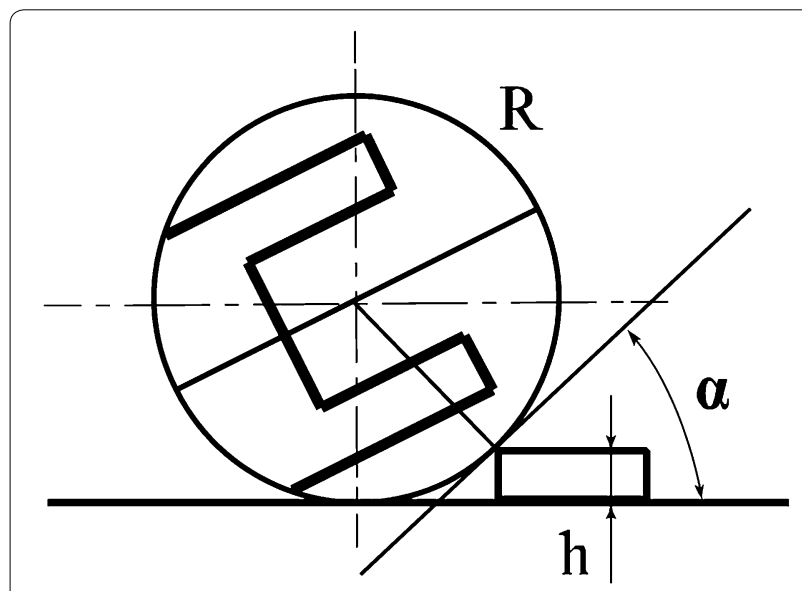

Fig. 14 Defining the slope 


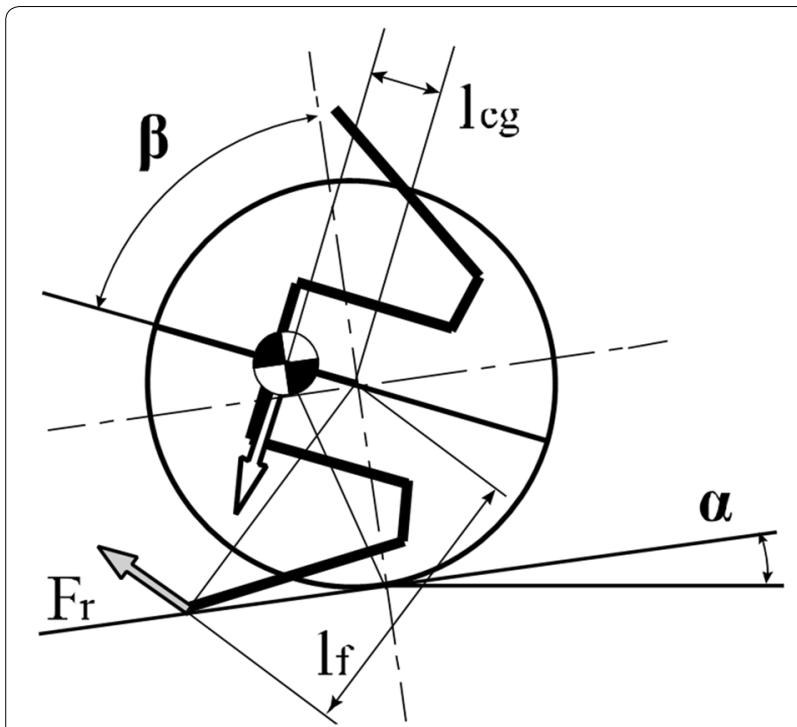

Fig. 15 Analysis of rising motion

uneven ground, such as on rubble (Fig. 14). To estimate the ability to rise on uneven terrain, we define the influence of rubble contacts as slope inclination. Assuming that the height of the block to be contacted is $h$, the angle of the slope is $\alpha$ from the Eq. (1). $R$ is the radius of the spherical outer shell: $100[\mathrm{~mm}]$.

$$
\alpha=\cos ^{-1}(1-h / R)
$$

To calculate the conditions under which rising motion can be executed, the analysis was carried out (Fig. 15). It is assumed that QRoSS V is placed on the slope of the angle $\alpha$ inclined by $\beta$. To rise in the direction of climbing the slope, as shown in the Eq. (2), the moment generated at the leg tip needs to be larger than the moment of rotating the outer shell where the center of gravity occurs.

$$
F_{r} \cdot l_{f}-\mathrm{Mg} \cdot \sin (\alpha+\beta) \cdot l_{c g}>0
$$

Here, it is assumed that the mass is $M: 1034$ [g], the gravitational acceleration is $g: 9.8\left[\mathrm{~kg} / \mathrm{s}^{2}\right]$, the distance from the center of the outer shell to the center of gravity is lcg: $20[\mathrm{~mm}]$, the rotation component of the reaction force with the ground generated at the toe is $F r$, the distance from the center of the outer shell to the feet is denoted by lf. Fr is determined by the output of the actuator whose torque is $13.6[\mathrm{kgf} \mathrm{cm}]$ and the weight of the leg is ignored at deployment. This model is assumed to be a static model and there is no slip between the outer shell and the slope. The range of $\beta$ is 0 [deg] to 157.4 [deg]. Figure 16 shows the results of this simulation. On the horizontal axis are

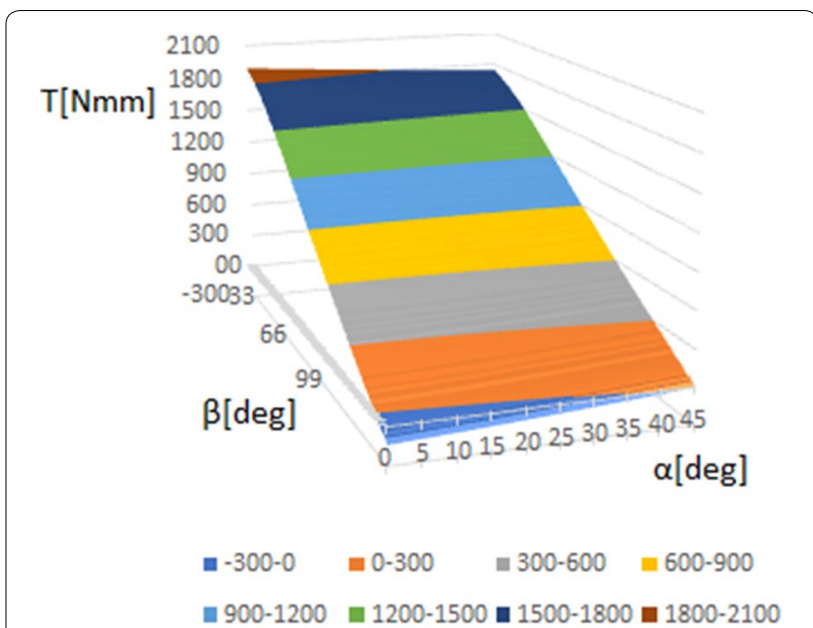

Fig. 16 Simulation results of rising motion

the angle $\alpha[\mathrm{deg}]$ of the slope and the incline $\beta[\mathrm{deg}]$ of the spherical shell; the vertical axis is the rotation torque $T[\mathrm{Nmm}]$ of the shell. From this result, in almost all areas that the incline $\beta[\mathrm{deg}]$ and the slope angel $\alpha[\mathrm{deg}]$, the QRoSS V can rise in the whole area because the actuator's torque is sufficiently large. However, it can rise to 20 [deg] from the experiment results of the real model of QRoSS V. Figure 17 shows the results of rising motion of the real model; the horizontal axis is time and the vertical axis is the rotation angle. Because the rotation angle is measured from the final posture after the rolling motion, the final arrival angle is $-180\{\mathrm{deg}]$ in this experiment. The orange line was tested on flat ground, and the blue line was on a slope of 20 [deg]. If the inclination exceeded 20 [deg], the outer shell and the toes slipped; it could not rise.

To calculate the conditions under which rolling motion can be executed, this analysis was carried out (Fig. 18). Because the rolling motion is performed by leg kick, the rolling continues by rotation of the center of gravity due to gravity after the legs have elongated. However, this model is assumed to be a static model and there is no slip between the outer shell and the slope, and the weight of the kicking feet is ignored. The condition of rotation is the Eq. (3),

$$
M g \cdot \sin (\beta-\alpha) \cdot l_{c g}>0
$$

The value of $\beta$ is 22 [deg]. Figure 19 shows the results of this simulation. The horizontal axis is the angle $\alpha$ [deg] of the slope, and the vertical axis is the rotation torque $T[\mathrm{Nmm}]$ of the outer shell. If $\mathrm{T}$ is a positive value, the rolling motion succeeds. If $\alpha$ is 22 [deg] or less, the rolling motion will succeed but if it is more, QRoSS V cannot rotate. When calculating $h$, which is the height of the block touched with the shell from this 


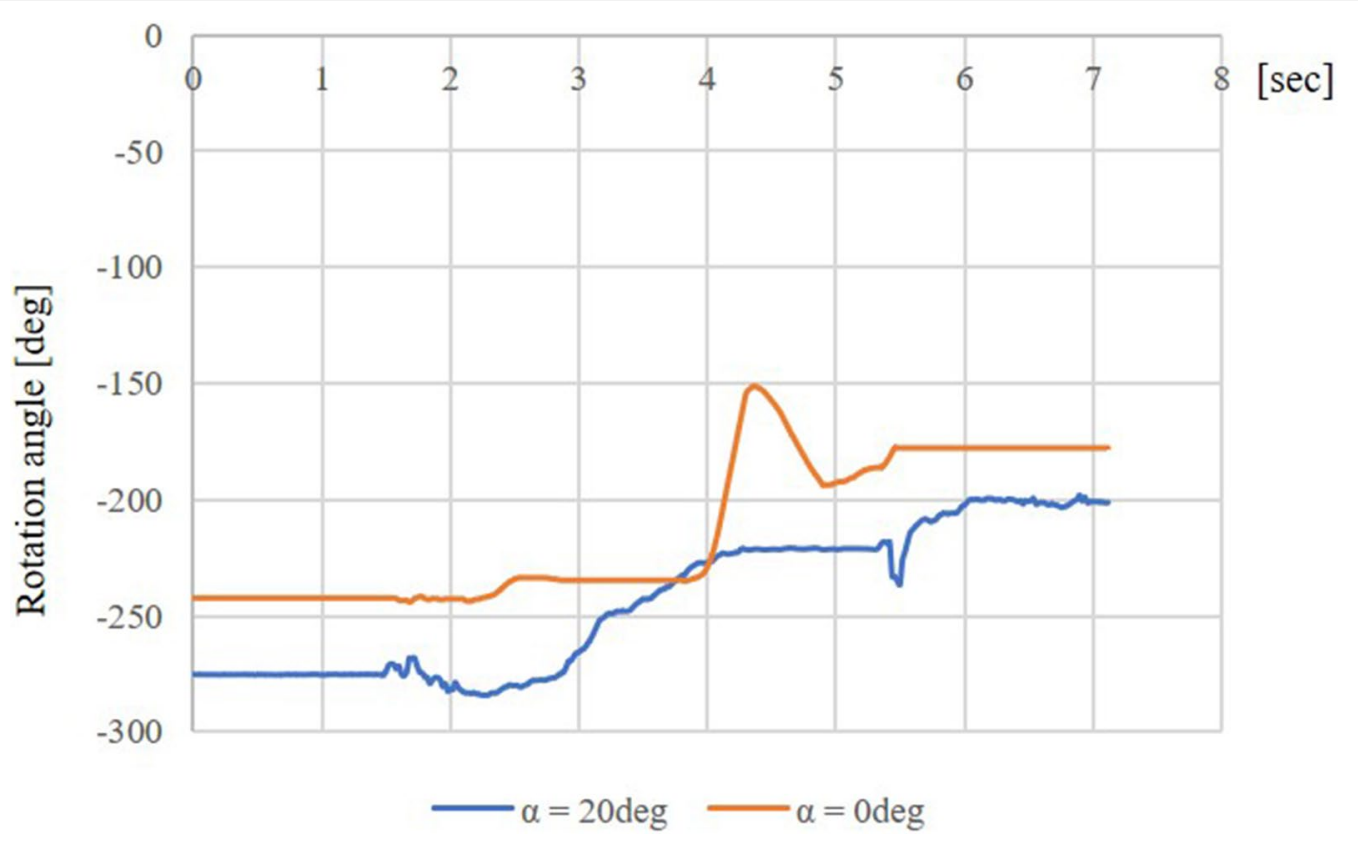

Fig. 17 Experiment results of rising motion of real model

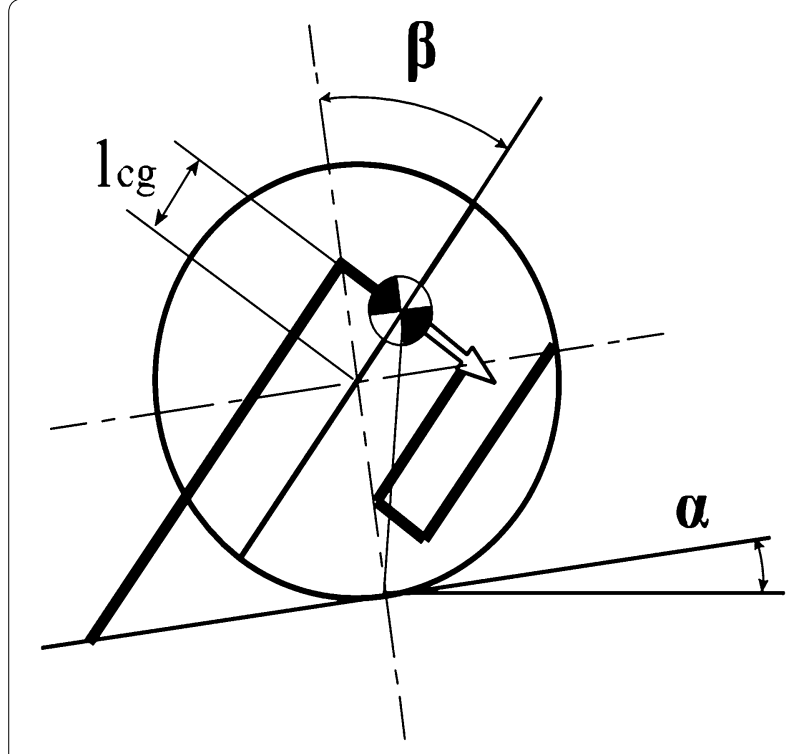

Fig. 18 Analysis of rolling motion

condition, h becomes 7.28 [mm]. Figure 20 is the result of the rolling motion of the real model, the horizontal axis is time and the vertical axis is the rotation angle. The rolling motion succeeded only at less than 10 [deg]. This is due to the occurrence of C.O.G movement in the outer shell by changing legs posture during rotation, and the spherical outer shell is deformed into an ellipsoid. The height: $\mathrm{h}$ from the experiment result is $1.6[\mathrm{~mm}]$.

\section{Performance tests and discussion}

Two performance tests were conducted to verify effectiveness of the improved design of QRoSS V; we discuss those results here. Although experiments were made using an external power cable and wired communication, the cables did not prevent mobility of the experimental robot.

\section{Walking performance}

As a verification of basic mobility, we conducted a walking experiment using the crawl gait. This gait is static walking, which is always stable walking by the support polygon. When the duty ratio of the gait was set at 75 [\%], QRoSS V did not fall over, but could not walk steadily. This is because the center of gravity position deviated from the support polygon and its stability margin is too low. On the other hand, by setting the duty ratio to 80 [\%], it could walk with a stable gait adding the state of four-point support. Its speed was $0.03[\mathrm{~m} / \mathrm{s}]$. The ability to move them four directions was confirmed: front, back, left and right, regardless of the direction of the body. Compared with QRoSS I, the maximum stride length of QRoSS V is 120 [mm] and bigger than QRoSS I whose stride is $35[\mathrm{~mm}]$. The stability margins [17] at that time are QRoSS V: $13.1[\mathrm{~mm}]$ and QRoSS I: $4.3[\mathrm{~mm}]$.

\section{Climbing performance}

To verify the mobility of QRoSS V on uneven terrain, we conducted an experiment of the step, which was 


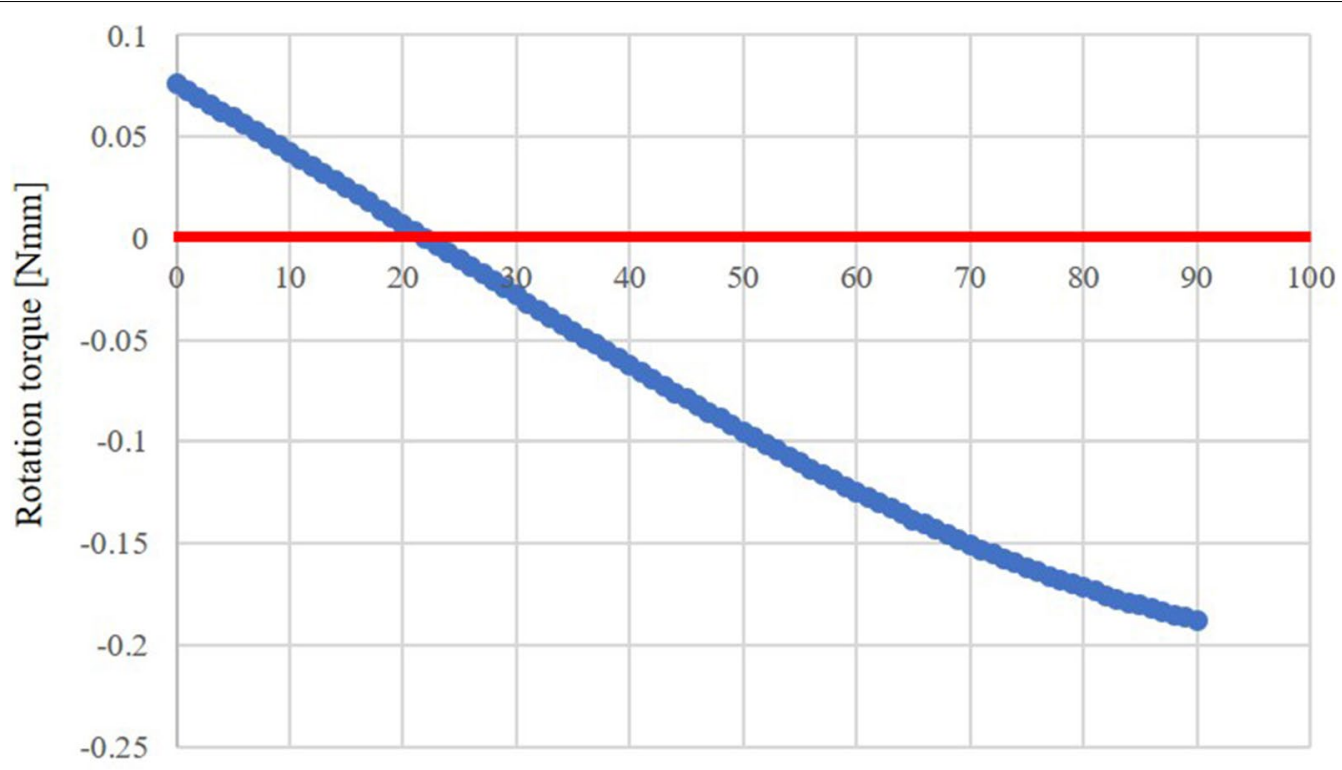

Slope angle [deg]

Fig. 19 Simulation results of rolling motion

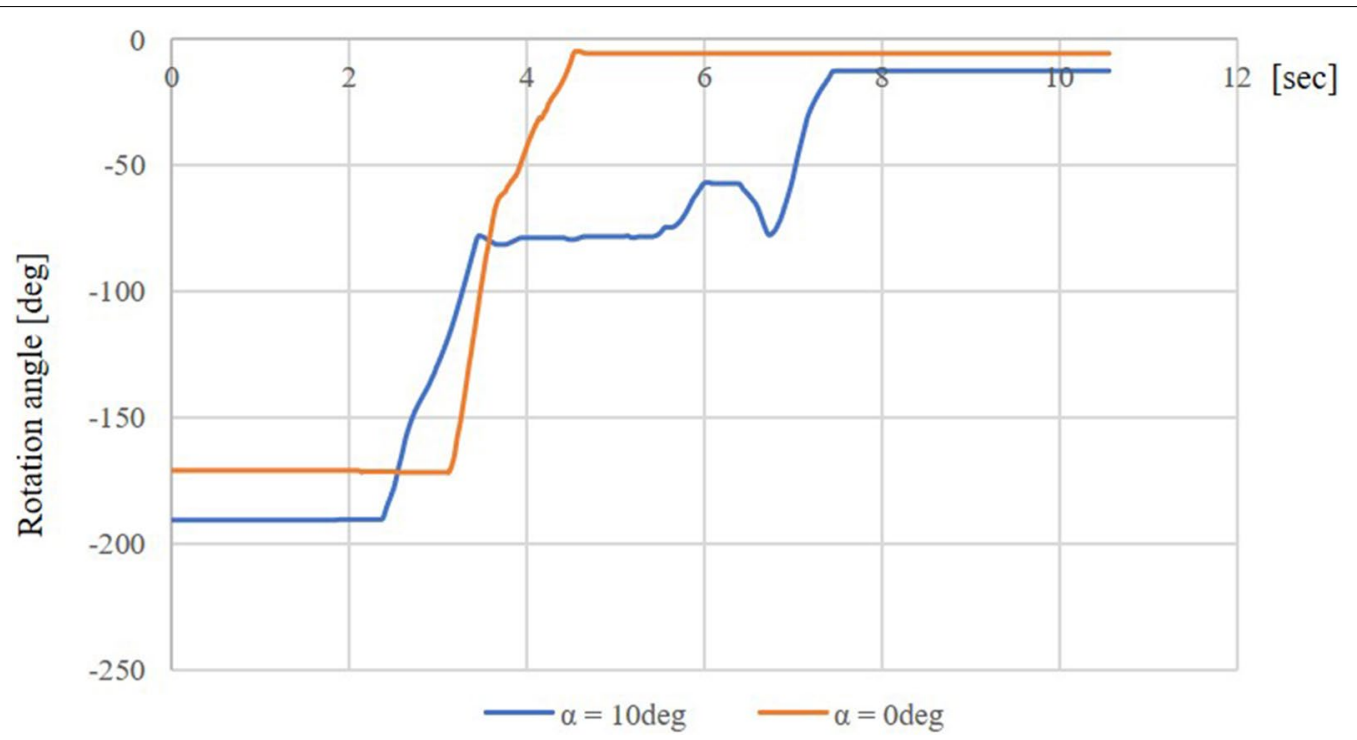

Fig. 20 Experiment results of rolling motion of real model

standardized. The experimental situation is shown in Fig. 21. This experiment is not done by dynamic gait. This is because it is better not to destroy rubble in actual rescue operations. The climbing motion of the robot in this experiment was generated beforehand based on kinematics. First, the front two legs were put on the step as in No.3 to No.5. Next, the main body moved over the step and its center of gravity moved above the step as in
No.6 to No.7. After progressing to the distance where the rear leg can be placed on the step, the rear leg was lifted to complete the series motion. From this result, it was confirmed that QRoSS V can climb over a step of up to 120 [mm], which is 60 [\%] of the outer shell without the shell touching a corner of the step. Figure 22 shows the movement trajectory of the foot when climbing a step with a height of $140 \mathrm{~mm}$. When climbing 


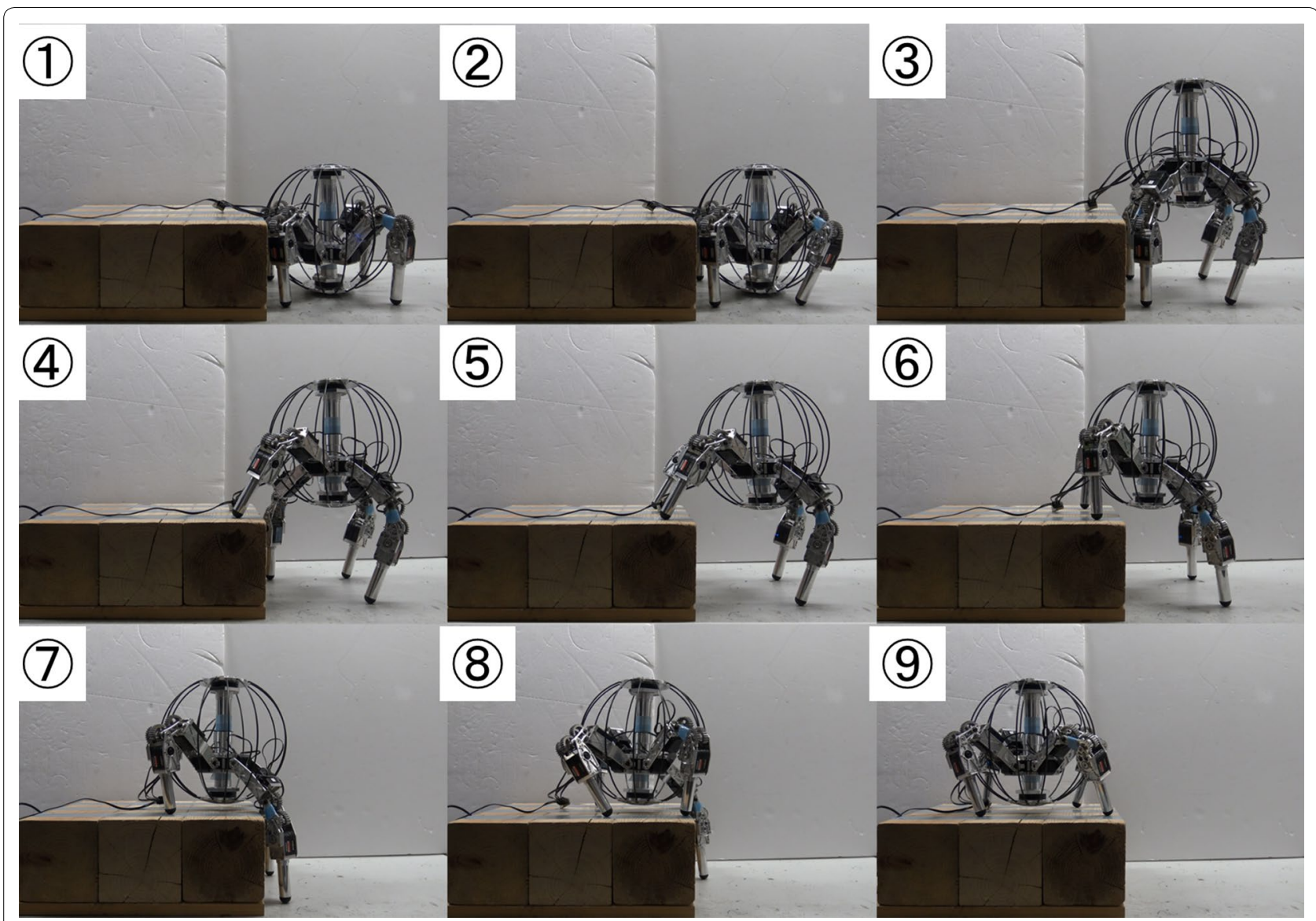

Fig. 21 Climbing over a step (height is $120 \mathrm{~mm}$ )

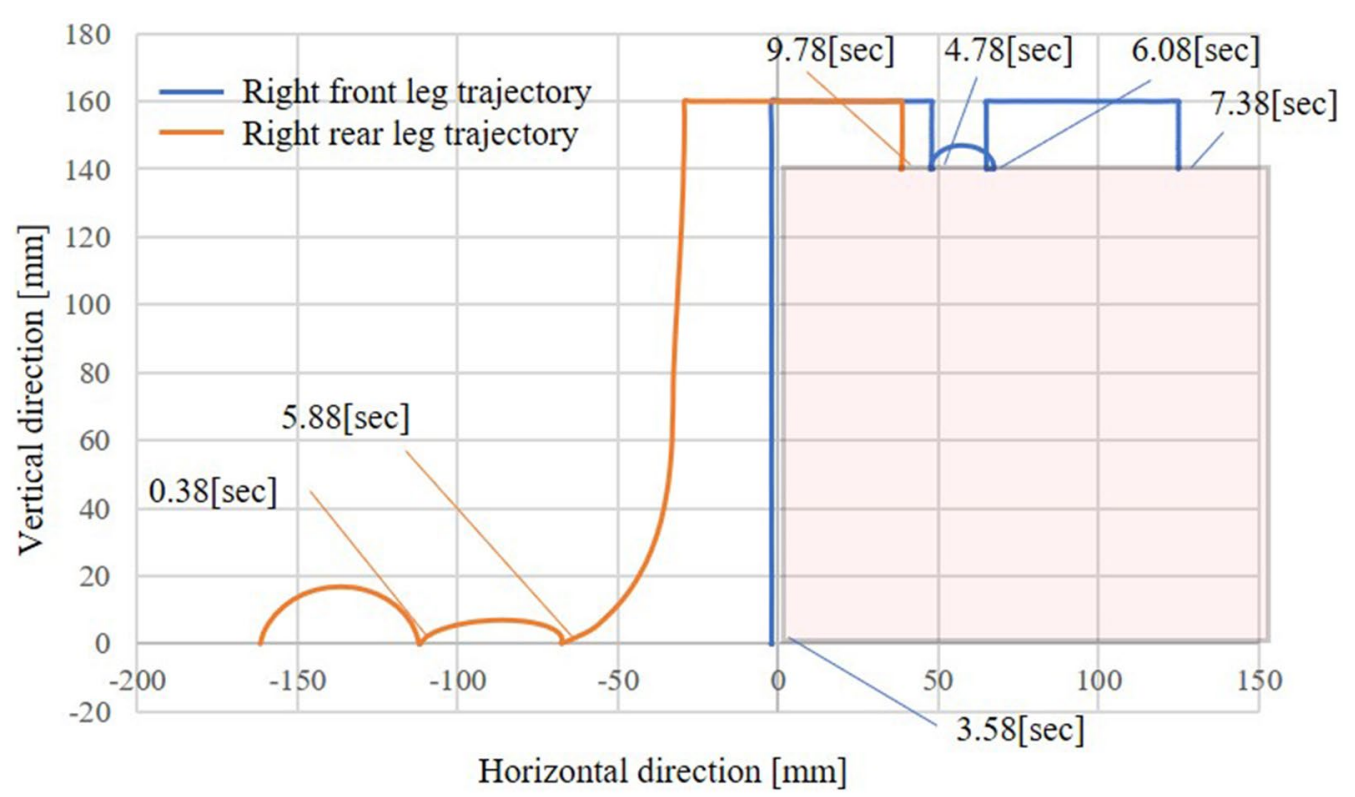

Fig. 22 Foot trajectory of climbing over a step (height is $140 \mathrm{~mm}$ ) 


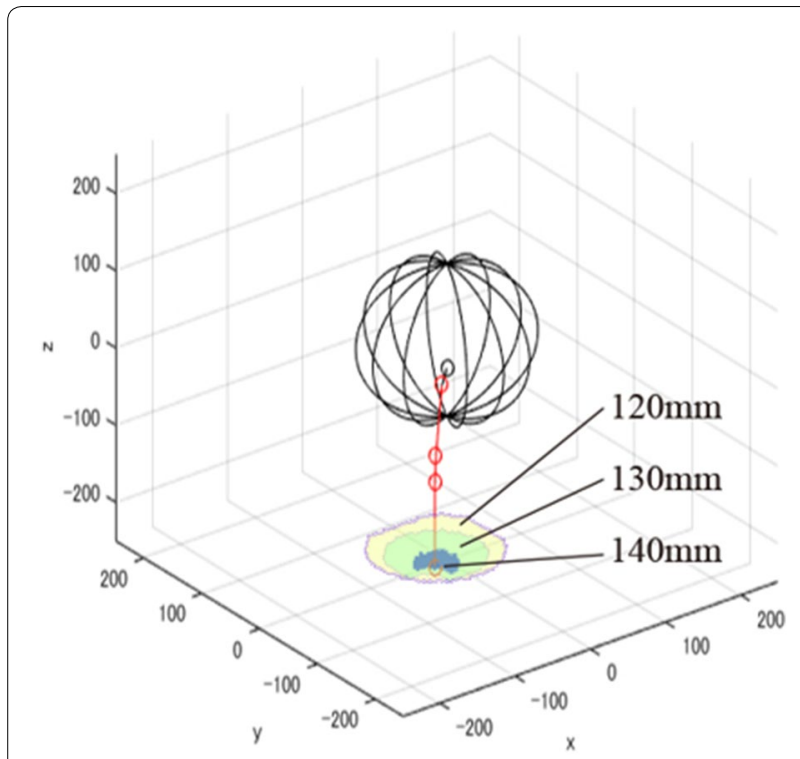

Fig. 23 Work ranges of foot at distances from the spherical outer shell

Table 2 Comparison of step-leg length ratio

\begin{tabular}{lllll}
\hline & $\begin{array}{l}\text { TITAN XII } \\
\text { [15] }\end{array}$ & $\begin{array}{l}\text { Little Dog } \\
\text { [16] }\end{array}$ & $\begin{array}{l}\text { QRoSS II } \\
\text { [11] }\end{array}$ & $\begin{array}{l}\text { QRoSS } \\
\text { V }\end{array}$ \\
\hline $\begin{array}{l}\text { Leg length [mm] } \\
\begin{array}{c}\text { Overtable step } \\
\text { height [mm] }\end{array}\end{array}$ & 1130 & 167 & 290 & 220 \\
$\begin{array}{c}\text { Step-leg length ratio } \\
\text { [\%] }\end{array}$ & 49.6 & 45 & 100 & 120 \\
\hline
\end{tabular}

this height, the range in which the toes can move is reduced, stepping actions are required. In Fig. 23, the leg trajectories are calculated by forward kinematics; the distance from the outer shell and the work ranges of the foot are shown. QRoSS V can climb over a step with a height of $140 \mathrm{~mm}$ by moving the legs with the outer shell grounded on the step. However, because the work range of the foot is too small, it is necessary to step on the steps again and again, we believe practical mobility cannot be obtained. We also considered the gait using its outer shell as the contacting point with the step and stability during climbing became higher; however, the height of the step that could be overcome did not improve. Based on results of the climbing experiments at this time, we calculated a step-leg length ratio by dividing the step height that can be climbed by the leg length, and compared it with robots of previous research, which are the TITAN XII [14], the Little Dog [15] and QRoSS II [11] (Table 2). The actual height of the step that QRoSS V can climb over is not very high compared with previous research because its body is small. However, the step-leg length ratio is 54.5 [\%], which is the highest of all, so the climbing performance of QRoSS V is proved to be superior.

\section{Conclusion}

We proposed a quadruped walking robot (QRoSS) with a spherical shell and developed the fifth prototype model. QRoSS V is a transformable robot and can change from its storage state, in which four legs are stored in the spherical shell, to deploy the legs outside the shell. The shell not only absorbs external forces from all directions, but also improves mobile performance by virtue of its round shape. In this paper, we propose and develop QRoSS V with new mechanisms that offset the main frame of the leg's base and stand leg length to improve walking performance. The paper reports analyses and experiments of the rising operation of the QRoSS V and the performance experiment of climbing over a high step. From the analysis result of the rising operation, a series of the rising motion and the rolling motion is possible on slopes of up to 22 [deg]; however, experiments using an actual robot reveal the rising motion is up to 20 [deg] and the rolling motion is up to 10 [deg]. In the step climbing experiment, QRoSS V was able to climb a step of 140 [mm] height, showing higher stepping ability compared with other robots.

Acknowledgements

Not applicable.

Authors' contributions

Not applicable

Funding

This work was supported by JSPS KAKENHI Grant Number JP26420206 and Takano Science Foundation.

Availability of data and materials

Not applicable.

Ethics approval and consent to participate

Not applicable.

Consent for publication

Not applicable.

Competing interests

The authors declare that they have no competing interest.

Received: 18 October 2019 Accepted: 7 May 2020

Published online: 13 May 2020

References

1. Wooden D, Malchano M, Blankespoor K, Howardy A, Rizzi AA, Raibert M (2010) Autonomous Navigation for BigDog. IEEE International Conference on Robotics and Automation Anchorage Convention District

2. Bajracharya M, Ma J, Malchano M, Perkins A, Rizzi AA, Matthies L (2013) High fidelity day/night stereo mapping with vegetation and negative 
obstacle detection for vision-in-the-loop walking. In: IEEE/RSJ International Conference on Intelligent Robots and Systems (IROS)

3. Shiroma N, Chiu YH, Min Z, Kawabuchi I, Matsuno F (2006) Development and control of a high maneuverability wheeled robot with variablestructure functionality. In: Proc. IEEE/RSJ International Conference on Intelligent Robots and Systems(IROS2006), Beijing, October 9-15, 2006

4. Nagatani K, Kiribayashi S, Okada Y, Otake K, Tadokoro S, Nishimura T, Yoshida T, Koyanagi E, Hada Y (2011) Redesign of rescue mobile robot Quince -Toward emergency response to the nuclear accident at Fukushima Daiichi Nuclear Power Station on March 2011. In: Proceedings of the 2011 IEEE International Symposium on Safety,Security and Rescue Robotics

5. Arai M, Toshio T, Shigeo H (2004) Development of "Souryu-III": connected crawler vehicle for inspection inside narrow and winding spaces. In: Proc. of IEEE/RSJ Int. Conf. on Intelligent Robots and Systems (IROS), pp. 52-57

6. iRobot 510 PackBot (2017), available from http://www.irobot.com/dk/filel ibrary/pdfs/iRobot_PackBot_Scout.pdf. Accessed 14 Sept 2017

7. Ryuichi H, Yasuaki F, Hisanori A, Shigeo H. (2010) Development of trackchangeable quadruped walking robot TITAN X -design of leg driving mechanism and basic experiment. In: Proc. of IEEE/RSJ Int. Conf. on Intelligent Robots and Systems (IROS), pp 3340-3345

8. IEEE SPECTRUM, FirstLook: iRobot's New Throwable Baby Surveillance Bot (2017). http://spectrum.ieee.org/automaton/robotics/military-robots/ firstlook-irobot-new-throwable-baby-surveillance-bot. Accessed 14 Sept 2017

9. IEEE SPECTRUM, Boston Dynamics Sand Flea Robot Demonstrates Astonishing Jumping Skills (2017) https://spectrum.ieee.org/automaton/robot ics/military-robots/boston-dynamics-sand-flea-demonstrates-astonishin g-jumping-skills. Accessed 14 Sept 2017
10. Takeshi A, Kazuki O (2014) Design of Quadruped Walking Robot with Spherical Shell. In: Proc. of Int. Conf. on Design Engineering and Science, pp 116-121

11. Aoki T, Ito S, Sei Y (2015) Development of quadruped walking robot with spherical shell -mechanical design for rotational locomotion. In: IEEE/RSJ international conference on intelligent robots and systems (IROS)

12. K. Halvorsen: "Morphex III" (2017), http://blog.robotpark.com/innovation -contest-winner-hexapod-morphex-31007/, Accessed 14 Sept 2017

13. Bunathuek N, Saisutjarit P, Laksanacharoen P (2014) Design of a reconfigurable spherical robot II. In: Proc. of IEEE Cyber, pp 62-66

14. Kinjo T, Aoki T (2017) Realization of jumping motion for walking robot with spherical outer shell. In: The 4th international conference on design engineering and science, 242

15. Komatsu Hirone, Endo Gen, Hodoshima Ryuichi, Hirose Shigeo, Fukushima Edwardo $F(2015)$ How to optimize the slope walking motion by the quadruped walking robot. Adv Robot 29(23):1497-1509

16. Buchli J, Kalakrishnan M, Mistry M, Pastor P, Schaal S (2009) Compliant quadruped locomotion over rough terrain. In: Proc. of Int. Conf. on Intelligent Robots and Systems, pp 814-820

17. McGhee RB, Frank AA (1986) On the stability properties of quadruped creeping gaits. Math Biosci 3:331-351

\section{Publisher's Note}

Springer Nature remains neutral with regard to jurisdictional claims in published maps and institutional affiliations.

\section{Submit your manuscript to a SpringerOpen ${ }^{\odot}$ journal and benefit from:}

- Convenient online submission

- Rigorous peer review

- Open access: articles freely available online

- High visibility within the field

- Retaining the copyright to your article

Submit your next manuscript at $\boldsymbol{\nabla}$ springeropen.com 